\title{
APUNTES PARA UN NUEVO DICCIONARIO DE ESCRITORES SEVILLANOS
}

\author{
Aurora Domínguez Guzmán
}

Una de las tareas pendientes en el campo de la investigación que tiene nuestra Universidad, muy laboriosa, pero no por ello menos necesaria, es la de confeccionar un Diccionario bio-bibliográfico de escritores sevillanos actualizado y hecho con el mayor rigor científico posible.

Cuando se aborda cualquier trabajo de tema local o no, en el que entran en juego autores nacidos en Sevilla o en su provincia con anterioridad a este siglo, sobre todo si no son figuras de primera línea, comienza un proceso de todos conocidos. Se acude en primer lugar a esa especie de Biblia que viene a constituir para los estudiosos el Diccionario que en su día hizo Méndez Bejarano ${ }^{1}$, y después al Manual de Palau ${ }^{2}$. En el caso de que los autores a tratar sean anteriores al siglo XIX, resulta obligada la consulta de los trabajos de Simón Díaz y Aguilar Piñal ${ }^{3}$ dentro de los límites que por ahora tienen, así como la Tipografía Hispalense de Escudero ${ }^{4}$. A partir de ahí, se sientan las bases para el trabajo y comienza el tradicional peregrinaje documental y bibliográfico. Si el autor no es muy conocido y pertenece a época más reciente, el proceso a su vez, aunque parezca paradójico, se suele complicar más por la falta de trabajos del rigor de los de Simón Díaz y Aguilar Piñal que hemos citado.

1 Mario Méndez Bejarano, Diccionario de escritores, maestros y oradores naturales de Sevilla y su actual provincia (Sevilla: Tipografía Gironés, 1922-1925), 3 vols.

2 Antonio Palau y Dulcet, Manual del librero hispano-americano (Barcelona: 1948-1977), 2. ed. corregida y aumentada, 28 vols.

3 José Simón Díaz, Bibliografía de la Literatura Hispánica (Madrid: C.S.I.C., 1950-1984), 14 vols. En publicación (autores comprendidos entre las letras A-M). Francisco Aguilar Piñal, Bibliografía de autores Españoles del siglo XVIII (Madrid: C.S.I.C., 1981-1986), 4 vols. En publicación (autores comprendidos entre las letras A-K).

4 Francisco Escudero y Perosso, Tipografía Hispalense. Anales bibliográficos de la ciudad de Sevilla desde el establecimiento de la imprenta hasta finales del siglo XVIII (Madrid, Sucesores de Rivadeneyra, 1894). 
Buena parte de ese proceso podría paliarse con un nuevo Diccionario en el cual, además del ensamblaje de las noticias de Méndez Bejarano con las aportaciones bibliográficas de los referidos catálogos y la incorporación obligada de autores más recientes, habría que tener muy en cuenta otros factores:

1.) Los numerosos estudios, monográficos o no, que se han ido sucediendo sobre escritores sevillanos, en especial cuando estos son de primera línea, e incluso de segunda, y que habría que inventariar en cada caso.

2.) Otros repertorios biográficos anteriores y posteriores al de Méndez Bejarano, ya sean de autores locales o españoles. Entre estos, sigue resultado fundamental el de Rodríguez Marín ${ }^{5}$ con un alto índice de escritores sevillanos. Muy útiles también son aquellos que se han publicado relativos a los miembros de determinadas órdenes religiosas.

3.) Que además de la consulta de los catálogos bibliográficos citados y algunos más ya clásicos como los de Nicolás Antonio, Gallardo, Salvá, el de la Hispanic Society of America, etc., no hay que olvidar que existen otros no tan conocidos, bien por ser más limitados o por su reciente aparición, que registran la existencia de obras, en ocasiones de gran interés, de escritores sevillanos de las que no teníamos ni tan siquiera noticia, o sólo esta, pero no de ejemplares conservados.

4.) También los fondos aún por explorar, o que lo han sido de forma insuficiente, de archivos y bibliotecas sevillanos. Un ejemplo de ello son los de la Biblioteca Arzobispal, muy ricos, pero hoy por hoy, y mucho nos tememos que durante bastante tiempo, tan mal catalogados y que, no olvidemos fueron un verdadero filón de noticias para Alonso Morgado.

5.) Que el trabajo a llevar a cabo habría de ser obviamente de equipo e interdisciplinario dada su amplitud y diversidad de campos (histórico, literario, científico...), apoyado a su vez en un fuerte aparato bibliográfico.

Sin duda, toda esta ingente labor se vería especialmente favorecida, dada su naturaleza, con las nuevas técnicas informáticas.

Se trata por supuesto de una ardua empresa pero que está ahí, invitándonos a emprenderla a través de un abundantísimo material, aunque su consecución sea a muy largo plazo, y ya sabemos que son los resultados rápidos los que cada día cuentan con mayor atractivo en el campo de la investigación como en tantas otras cosas.

Las aportaciones que aquí recogemos, aunque modestas, son una prueba de la utilidad de dicho trabajo, pues ni siquiera responden a esa necesaria y deseable yuxtaposición de datos tomados de Méndez Bejarano, Palau, Simón Díaz, etc., como es la cadena habitual. Son noticias de escritores sevillanos de los Siglos de Oro, en especial del XVII, de muy escasa relevancia en muchos de los casos aunque no lo olvidemos, jugaron un papel, pobre si se quiere pero muy destacado en la vida cultural de la Sevilla del Barroco. Sus sermones, su fervor mariano, su celo moralista... cobran en las prensas locales el más fiel reflejo. Si en el siglo XVI la capital andaluza fue el primer centro productor de libros del país, siguién-

5 Francisco Rodríguez Marín, Nuevos datos para las biografías de cien escritores de los siglos XVI y XVII (Madrid: Tip. de la «Revista de Archivos, Bibliotecas y Museos», 1923). 
dole en importancia Barcelona y Salamanca, en el XVII, aunque no tenemos datos comparativos, continuó manteniendo un altísimo nivel de producción.

Las noticias son básicamente bibliográficas y en algunas ocasiones también biográficas, pues gracias a la estética imperante, las obras como es sabido ven la luz con amplísimos títulos y verdaderos curricula de sus autores en las portadas ${ }^{6}$. Es más, estos apuntes que traemos aquí, vienen una vez más a demostrarnos lo mucho que hay aún por hacer en materia bibliográfica ya que por lo general proceden de los fondos antiguos de la Biblioteca Universitaria de Sevilla, de los mejores conocidos por su importancia y, sin embargo, algunos de sus textos han escapado a las sucesivas revisiones sistemáticas de que han sido objeto.

A continuación, damos esas nuevas noticias de escritores sevillanos siguiendo un orden alfabético de los mismos.

BRAVO DE LAGUNA, Juan: Un problema se nos plantea con este autor. Según Méndez Bejarano murió en 1631 y según Simón Díaz (VI, pág. 656) antes de 1635 , y no fue, como figura en el texto que más abajo señalamos, Familiar del Santo Oficio, cargo que sí ostentó un sobrino suyo llamado Andres Bravo de Laguna. ¿Se llamó este en realidad Juan o Juan Andrés? Desde luego en el texto siguiente, no registrado de los repertorios bibliográficos, figura como autor Juan Bravo de Laguna:

Parecer del R. P. Maestro -, Calificador del Santo Oficio de la Inquisición, acerca de la pena que merece el blasfemo que fixò el cartel (Sevilla, Francisco de Lyra, 1640. 28'5 cms. 2 hs.). Sevilla. Bib. Universitaria, 111/142(9). El suceso ocurrió en Granada la noche del Jueves Santo de 1640 y el cartel contenía blasfemias relativas a Cristo y la Virgen.

CALDERA DE HEREDIA, Gaspar. Entre las varias obras que vieron la luz de tan famoso médico sevillano, sólo Goldsmith ${ }^{7}$ recoge la siguiente:

Vista, visita y reconocimiento del cuerpo del venerable siervo de Dios el rey don Fernando el Santo. (¿Sevilla? ¿1668? Fol.). Londres, British Museum, T. 16*. (36).

CÁRDENAS, Juan de: Para este jesuita, primer biografiador de D. Miguel de Mañara, hay que tener muy en cuenta el excelente estudio del P. Granero sobre dicho personaje ${ }^{8}$. En él, entre otras cosas, da a conocer en la Bibliografía varios textos de Juan de Cárdenas que estaban inéditos.

En la Bib. Arzobispal de Sevilla, con la signatura Est. 38, n. 103 (31), se encuentra una epístola del jesuita Martín de Zuaznabar, sin lugar de impresión

6 Como muestra de ello, véase más adelante la obra que citamos de Fray Juan de Mena.

7 V. F. Goldsmith, A short title catalogue of spanish and portuguese books 1601-1700 in the Library of British Museum (Folkestone and London: Dawsons of Pall Mall, 1974), C, 48.

8 Jesús M. Granero, Don Miguel Mañara Leca y Colona Vicentelo (Un caballero sevillano del siglo XVII). Estudio biográfico (Sevilla: Artes Gráficas Salesianas, 1963). Obra de difícil consulta, es una fuente valiosísima de noticias sobre la Sevilla del siglo XVII acompañada de un abundante aparato documental y bibliográfico. Con posterioridad, el padre Granero en edición de bolsillo ha publicado una nueva biografía de D. Miguel de Mañara. 
ni año pero fechada en Sevilla en 1684, sobre la muerte y virtudes del Padre Juan de Cárdenas que no hemos visto citada en ningún repertorio bibliográfico.

CARO DE MALLÉN, Ana. Sobre su lugar de nacimiento existen dudas ${ }^{9}$ aunque Méndez Bejarano ni siquiera se las plantea. El hecho de que en las obras de esta escritora nunca aparezca como «natural de la ciudad de Sevilla», fórmula muy común en tales casos, y en una ocasión como "vezina» ${ }^{10}$, nos parece un argumento más, nimio si se quiere, para no considerarla sevillana.

Soló Simón Díaz (VII, 5.110), sin referencia de ejemplar, da noticia de la obra que a continuación recogemos y que hemos localizado en la Bib. Universitaria de Sevilla con la signatura 86-A/85(2):

Relación en que se da quenta de las grandiosas fiestas, que en el Conuento de N. P. S. Francisco de la Ciudad de Seuilla se an hecho a los Santos Martires del Iapon. Compuesta en Octauas por Doña - . (Sevilla, Pedro Gómez [de la Pastrana], 1628, 13'5 cms. 8 hs.).

DELGADO Y BUENROSTRO, Antonio. Aunque Méndez Bejarano cita, sin señalar localización de ejemplar, la obra que seguidamente incluimos, ésta no aparece recogida en los repertorios bibliográficos,

Demostración alegórica Del esclarecido Patriarca Señor San Josef, y del SS. Sacramento precisamente descvbierto En la magestuosa fiesta anual, que le celebra el Illustrissimo Cavildo Ecclesiastico de la Puebla de los Angeles, en su Iglesia Cathedral... (Sevilla, Tomás López de Haro, 1680. 19 cms. 24 págs.). Sevilla. Bib. Universitaria, 113/19(6).

ESCALANTE, Fernando de. Una falsa edición hemos visto registrada de una obra suya:

Clipeus concionatorum verbi Dei in quo sunt sculptae omnes visiones symbolicae, et signa realia veteris Testamenti... (Sevilla, Gabriel Ramos Bejarano, 1611. Fol.). Londres British Museum, 3166. g. $3^{11}$.

Sin duda, se trata de la edición de 1612 que hemos examinado en la Bib. Universitaria de Sevilla cuya fecha de impresión aparece confusa pero que sus preliminares no dejan lugar a dudas. Precisamente, Gestoso dio a conocer un documento de 1610 sobre la impresión de la obra en el que figuran el autor y el tipógrafo $^{12}$.

9 Vid. Manuel Serrano y Sanz, Apuntes para una biblioteca de escritoras españolas (Madrid: 1903-1905. 3 vols.) I, págs. 177-216.

10 Relación de la grandiosa fiesta, y octava, qve en la Iglesia parroquial de el glorioso san Miguel de la Ciudad de Seuilla, hizo don Garcia Sarmiento de Sotomayor, Conde de Saluatierra... Por doña -, vezina de la dicha Ciudad... (Sevilla, Andrés Grande, 1635. 4\% 16 hs.). El título completo en el que podemos leer «vezina» solo aparece precisamente en Serrano y Sanz, Apuntes..., op. cit., pág. 214.

11 V. F. Goldsmith, A short title catalogue..., op. cit., E, 71.

12 José Gestoso y Pérez, Noticias inéditas de impresores sevillanos (Sevilla: 1924), pág. 145. 
FIGUEROA, Francisco de. Otro de los tantos médicos como florecieron en la Sevilla del siglo XVII y del que nos ha quedado una producción impresa considerable. En uno de sus trabajos: Aciam, de qua loquitur Celsus cap. XXVI, lib $V$ filum semper, acum nunquam significare. In fibulationem, et suturam... (Sevilla, Simón Fajardo, 1634), indica haber publicado en romance un año antes el mismo. Lo hemos localizado en el British Museum con la signatura 593. h. 17. $(122)^{13}$.

FLORINDO, Andrés. De este médico sólo son conocidas dos obras ajenas a su profesión sobre su ciudad natal, Ecija. En la Bib. Universitaria de Sevilla, con la signatura 112/1(4), se encuentra la siguiente:

Epistola familiaris, in Arte Medica apprime necessaria, de venae sectione, in morbi statu... (Sevilla, Juan de León, 1607. 19 cms. 8 hs.).

En la obra figura ya como médico que ejerce en Ecija y se dice que es discípilo del célebre Doctor Juan de Luna Vega a quien dirige la epístola.

GÓMEZ DE ROJAS, Alonso. En la Bib. Universitaria de Sevilla, con las signaturas 113/15(13) y 112/131(21) respectivamente, se encuentran sus dos siguientes sermones que no figuran en los repertorios bibliográficos:

Sermon fvnebre a onras de la venerable madre Francisca Dorotea, fundadora, i Priora del Convento de Dominicas Descalças de nuestra Señora de los Reyes, de la Ciudad de Sevilla... (Juan de Cabrera, 1624. 19 '5 cms. 14 fols.).

Sermon primero en el solemne Octavario que celebro, el gran monasterio, del Carmen de Sevilla, en la Canonización de San Andres Corsino... en 16 de Setiembre de 1629... (Sevilla, Luis Estupiñán, 1630. 19'5 cms. 14 fols.).

Localización de otras obras de Gómez de Rojas:

Relación de la santa muerte, suntuoso entierro, $i$ solenissimas onras del venerable... Fernando de Mata, Clerigo Presbitero, natural... de Sevilla... (Sevilla, Alonso Rodríguez Gamarra, 1612. $30 \mathrm{cms} .4$ hs.). Madrid. Academia de la Historia, Jesuitas, 132, n. 21 y Sevilla. Bib. del Palacio Arzobispal, Est. 33, n. 209 bis $(40)^{14}$.

Sermon, que predico el Ldo - . En el segundo Otavario (en honor de la Inmaculada), que celebró la insigne Cofradia de los Nazarenos... En sv Iglesia de San Antonio Abad... de Seuilla... (Sevilla, Francisco de Lyra, 1617. 20 '5 cms. 12 fols). Sevilla. Bib. Universitaria, 112/129(9).

HERRERA, Jerónimo de. Catedrático de la Universidad de Méjico, Protomédico de la ciudad y médico del Virrey y del Santo Oficio. No registrado por Méndez Bejarano. Escribió poesías y realizó varias traducciones de clásicos que figuran en la siguiente obra, cuyo prólogo también suscribe:

13 V. F. Goldsmith, A short title catalogue..., op. cit., F, 184.

14 La primera referencia de ejemplar se encuentra en Mercedes Agulló y Cobo, «Relaciones de sucesos» I: Años 1477-1619», en Cuadernos Bibliográficos, 20 (n. monográfico), C.S.I.C., 1966, n. 518. La sergiida referencia nos ha sido facilitada por Esperanza Bonilla Martínez. 
RIBERA FLOREZ, Dionisio. Relación historiada de las exeqvias funerales de la magestad del Rey D. Philippo II... Hechas por el Tribunal del Santo Officio de la Inquisición desta Nueua España... declarandose las Figuras, Letras, Hierogliphicos, Empressa, y Diuisas, que en el Tumulo se pusieron... (Méjico, Pedro Balli, 1600. 21 cms. 26 hs. +187 fols. +5 hs.). Existe ejemplar en Madrid en la Bib. Nacional, R-4.981.

JIMÉNEZ MELERO, Miguel: Vid. MELERO JIMÉNEZ, Miguel.

LEÓN GARAVITO. Con estos apellidos figuran cuatro escritores del siglo XVII, Francisco, Melchor, Jacinto y Rodrigo. Méndez Bejarano da noticias de Francisco y Melchor, sin especificar si existió algún vínculo familiar entre ellos. Se extiende sobre todo en el primero por haber contraido matrimonio, en segundas nupcias, con la poetisa sevillana Dña. Feliciana Henríquez de Guzmán. De Jacinto y Rodrigo no tenemos noticia de que hubiesen nacido en Sevilla, pero una de las obras que se conservan del segundo parece indicar cuando menos, cierta vinculación con la ciudad ${ }^{15}$.

También aparece en 1635 un Andrés de León Garavito que dirige al Monarca un amplio Memorial sobre la necesidad de reformar el gobierno en ciertas provincias de las Indias ${ }^{16}$. ¿Existió algún lazo entre todos ellos? En todo caso, lo que sí es evidente es la presencia de estos apellidos en América pues, aparte del Memorial y la obra de Jacinto ${ }^{17}$ que así parecen indicarlo, vemos allí a dos destacadas figuras: Juan Sebastián de León Garavito, que fue Obispo de Guadalajara, bien entrado el siglo XVII, y Fray Ignacio de León Garavito, dominico que a mediados del XVIII ocupó la primera cátedra de Matemáticas de la Universidad de San Felipe de Santiago de Chile ${ }^{18}$.

LUJÁN, Pedro de. Destacado humanista del siglo XVI y modesto impresor, no ha sido tratado con la atención que requiere. Méndez Bejarano da pocas noticias sobre él y equivocadas, pues indica que nació en el siglo XVII y, paradójicamente, señala la edición de sus Coloquios matrimoniales de 1579 hecha en Zaragoza. La primera vez que vio la luz esta notable obra fue, como es sabido, en

15 Afecto amoroso de... En los Geroglificos, Qvartetas, Dezimas y otros versos diferentes que escrivio a la gran fiesta que celebró la calle de Gallegos (actual Sagasta), en los desagravios de Maria santissima. Año de 1640. (s. 1., s. i., s. a. 10 hs. 20 cms.). Madrid. Academia de la Historia, 9-17-4-3.541. En José Simón Díaz, Impresos del siglo XVII. Bibliografía selectiva por materia de 3.500 ediciones príncipes en lengua castellana (Madrid: C.S.I.C. 1972), n. 342.

16 Palau, Manual del librero..., op. cit., VII, 135.591.

17 Relación de la Salvd milagrosa que dio el Bienaventurado Stanislao Kostka. Novicio de la Cía. de Jesús, a otro Novicio de la misma Cía... en la Casa de Provacion de San Antonio Abad, de la Ciudad de Lima, el día 13 de Noviembre de el año de 1673... (S. i., 1674. 6 hs. 4.). Madrid. Academia de la Historia, 9-17-3-3.487.

18 Fray Antonio de Ezcaray en la Introducción de su curiosa obra, Vozes del dolor, nacidas de la mvltitud de pecados, que se cometen por los trages profanos, afeytes, escotados, y culpables ornatos... (Sevilla, Tomás López de Haro, 1691), nos da buena cuenta del celo moralista del Obispo. Del segundo, Fray Ignacio de León Garavito, tomamos la noticia de Francisco Esteve Barba, Cultura virreinal (Barcelona: Salvat, 1965), págs. 296 y 812. 
Sevilla en 1550 y tuvo extraordinaria fortuna, pues muchas ediciones le sucedieron y pasó incluso a traducirse al italiano. En cambio, Rodríguez Marín nos proporciona nueve documentos muy interesantes para su biografía tomados del $\mathrm{Ar}$ chivo de Protocolos sevillanos ${ }^{19}$.

Aquí queremos poner de relieve la vinculación de este humanista con los libros de caballerías que no ha sido destacada; compuso el duodécimo libro de $A m a$ dís y se le atribuye Lepolemo.

LUNA VEGA, Dr. Juan de: Numerosos trabajos de medicina dio a la imprenta este famoso doctor natural de Marchena, siendo los más conocidos sus Exercitaciones Medicas. Teníamos como su obra más antigua impresa, un opúsculo de $1605^{20}$. Sin embargo, en la Bib. Universitaria de Sevilla en un volumen de varios con la signatura 100/99, hemos visto dos opúsculos suyos impresos por Clemente Hidalgo en 1600 y $1602^{21}$. También en ese volumen de varios, en primer lugar se halla un curioso texto sobre la legitimidad del doctor, hijo de Juan de Luna y Marina González de Vega, ambos de Marchena, con el proceso del pleito en contra de tal legitimidad que interpuso Francisca Muñoz y fue continuado por los hijos que esta tuvo con el padre de Juan de Luna Vega, pleito que se fue fallando siempre a favor de éste.

MATA, Fernando de: «De pocos hombres se habrán escrito tantas biografías como del venerable P. Mata» nos dice Méndez Bejarano, pero sin citar ninguna. Al menos impresa en el siglo XVII, no conocemos más que la aparecida el mismo año de su muerte, 1612, hecha por Alonso Gómez de Rojas y que citamos en el lugar correspondiente a este autor.

MELERO JIMÉNEZ, Miguel: No aparece recogido por Hernández Morejón ${ }^{22}$ ni por Méndez Bejarano. Sin embargo, todo parece indicar que no es otro que el Miguel Jiménez Melero que cita el primero y a quien remite el segundo. El trasvase de apellidos se debe a que así sucede en la obra impresa de este doctor de 1706, Tractatus de generatione et corruptione... De igual forma lo vemos en otra de 1707 que registra Aguilar Piñal ${ }^{23}$, cuyo título, mucho más explícito, nos despeja toda posible duda, El sol de la médica verdad... Desea manifestar las luzes de la verdad en el hecho, derecho y medicina Don Miguel Jiménez Melero,

19 Nuevos datos..., op. cit. 517-20.

20 Apologia... contra vna respvesta, o defensa, que el P. M. Iuan de Pineda... embio al Dotor Francisco Ximenez Guillen. (Sevilla, Clemente Hidalgo, 1605. 19 cms. 2 hs. + 36 págs.).

21 Son respectivamente: Excelentissimi Principis Dvcis Methimnae Sidoniae, a cubiculo quondam medico. Iam Hispalensi doctissimo Doctori Antonio Marquez, suus Doctor Luna Vega Medicus Hispalensis. (29'5 cms. 4 hs.). 111/99(7) y Ivdicivm Doctoris —... de Discurso in mittendo Sanguine pro Pestilentibus inflamationibus a parte his correspondenti, edito ab eruditissimo Philosopho, Medicoque. (29'5 cms. 3 hs. +1 h. en b.). 111/99(3). El proceso del pleito en 111/99(1).

22 Antonio Hernández Morejón. Historia bibliográfica de la Medicina en España, (Madrid: Imprenta de la viuda de Jordán e hijos, 1842-52), 7 vols.

23 Bibliografía de autores españoles..., op. cit., IV, no 4.877. 
Socio Fundador de la Sociedad de Sevilla y Familiar del Santo Oficio. Efectivamente, es el famoso doctor Miguel Melero Jiménez, uno de los fundadores de la célebre Academia Sevillana de Medicina y así aparece en los documentos ${ }^{24}$, que fue Familiar del Santo Oficio y quien mantuvo una dura polémica con Alonso López Cornejo, otro destacado médico sevillano partidario de la medicina tradicional, sobre el ejercicio de la profesión, que ha quedado reflejada en las prensas locales. Una contrarréplica de Miguel Melero hemos visto en la Bib. Universitaria de Sevilla con las signaturas 111/90(19) y 110/148(10), que no aparece en los repertorios bibliográficos. Es la que sigue:

Propvgnacvlo de el Alegato jvridico a favor del derecho y costvmbre, que tienen a preceder en las consvltas los medicos revalidados mas antigvos de la Vniversidad de la civdad de Sevilla. Contra respvesta a la segvnda parte de la question, qve con impropriedad (sic) llaman. Medico legal; Pvblicada por el Dr. Don Alonso Lopez Cornejo. Le formaba, y escrivì D. -, Medico revalidado, y familiar del Santo Oficio. (Sevilla, Herederos de Tomás López de Haro, 1697. 28 '5 cms. 6 hs. +44 págs.).

MENA, Fr. Juan de: Carmelita, nacido en Ecija, no figura en ninguno de los trabajos hechos sobre autores sevillanos. Hay tres ejemplares de una obra suya en la Bib. Universitaria de Sevilla, 110/44(12); 113/60(26) y 113/15(3) que cita Simón Díaz (XIV, 4.774), cuyo amplio título nos aporta algunos datos de su vida. Dice así:

Desagravios de Maria Santissima. De los agravios hechos a sv imagen santa, dandola a las llamas el perfido herege Olandès: en la Fuerça de Cabo. Celebrados en el insigne Octauario, que... hizo el Conuento Grande... del Carmen de... Seuilla, a siete de Nouiembre de 1638. años. Por el R. P. M. -, Conuentual del dicho Conuento, y Cathedratico en propriedad (sic) de la Cathedra de Durando, de la Vniuersidad de Ossuna, y natural de la ciudad de Ecija... (Sevilla, Simón Fajardo, 1638. $19 \mathrm{cms}$. 22 fols.).

MUÑOZ, Andrés (Muñones el Bueno). De este Artillero mayor de la Casa de la Contratación de Sevilla, natural de Carmona, se tenía noticia de la rara y curiosa obra,

Instruccion y Regimiento para que los Marineros sepan usar de la Artilleria con la seguridad que conviene...

Gallardo (III, 3.181-2) cita dos ediciones, una de 1563 y otra sin año. En otros repertorios aparecen estas así como la sevillana de 1602 hecha por Fernando de Lara, de la que existe ejemplar de la Hispanic Society of America y otra de 1642 que registra Nicolás Antonio. Sin embargo, hemos visto en el catálogo de Goldsmith una nueva edición de Sevilla hecha por Tomé de Dios Miranda de $1678^{25}$,

24 Vid. Gabriel Sánchez de la Cuesta. «La Regia Sociedad de Medicina». Momentos estelares de la medicina sevillana (Sevilla, 1967), pág. 80.

25 A short title catalogue..., op. cit., M, 630. 
«sacado a luz por Roque Roman de Enche». Erróneamente en dicho catálogo figura como autor Andrés Muñoz de Pueno.

NÚÑEZ NAVARRO, Francisco. Según Méndez Bejarano, tan excelente predicador astigitano nació en 1588 pero no da más noticia suya a partir de 1615 en que predicó un sermón dedicado a la Purísima que fue dado a la imprenta ese mismo año. Sin embargo, en la Bib. Universitaria de Sevilla hay dos ejemplares de otro sermón que predicó bastantes años después y que no ha sido registrado, con las signaturas 113/86(2) y 113/19(7):

Sermon del Santissimo Sacramento. En la famosa fiesta de svs desagravios. Que celebró el insigne Clero, y Hermandad de la Ciudad de Ecija... (Ecija, Luis Estupiñán, 1635. 20'5 cms. 19 fols.).

Este sermón de «desagravios», como tantos otros que se pronunciaron en el país, estuvo motivado por un reciente suceso, la ofensa que en Flandes unos soldados del ejército francés hicieron al Santísimo Sacramento. También, gracias al amplio título de este sermón, sabemos que Francisco Núñez Navarro fue Vicario y Juez Eclesiástico de Ecija.

De su obra más conocida: Del precepto evangelico de perdonar i amar al enemigo... segvn el Capitulo Quinto de San Mateo... en la edición sevillana de 1618 hecha por Gabriel Ramos Bejarano, dicen Escudero y Palau que hay ejemplar en la Bib. Universitaria de Sevilla. En la actualidad desde luego no. En cambio sí se encuentra en la Bib. Pública de Córdoba con la sign. 10-104.

PEÑA, Fray Antonio de la. Méndez Bejarano, con ciertas reservas, atribuye a este sevillano una obra sobre la vida de Santa Catalina de Siena y otras santas vírgenes de la orden de Santo Domingo. En todo caso, su labor fue la de traducir del latín al castellano el texto de Fray Raimundo de Capua ${ }^{26}$.

PINEDA, Juan de. Entre su abundante producción impresa, Escudero, a quien remite Palau y seguramente copió Méndez Bejarano, recoge sin localización de ejemplar el texto que sigue:

Sermon a Ivezes y otros ministros de Ivsticia... (Sevilla, s. i., $1609.19 ' 20 \mathrm{cms}$. 20 fols.).

Hay ejemplar en Córdoba. Bib. Pública, 3-77.

PLATA, Fr, Sebastián de la. Sólo Palau (XIII, 228. 624-II) cita un sermón de este dominico no registrado en Méndez Bejarano. En el título se dice del autor que es natural de Sevilla y que pertenece Real Convento de San Pablo de esta ciudad, notas que se omiten en la cita de Palau. Tres ejemplares se conservan en la

26 Vid. Simón Díaz, José, Mil biografías de los Siglos de Oro (Índice Bibliográfico), Cuadernos Bibliográficos, 46, (N. monográfico), C.S.I.C., 1985, n. 188. Sólo en este repertorio, y en la misma referencia, hemos visto la cita, sin localización de ejemplar, de una edición sevillana de 1695 del texto de Capua traducido por Fray Antonio de la Peña. Existe en la Bib. Universitaria de Sevilla con la signatura 122/135. 
Bib. Universitaria de Sevilla de esta pieza oratoria: 113/60(16), 112/97(10) y $111 / 47(2)$.

Sermon predicado al Cabildo de la Santa Iglesia de Sevilla... en la solemnissima fiesta que celebro de la Presentación de la Virgen... 21. de Noviembre de 1622. Por el Padre -... (Sevilla, Francisco de Lyra, 1623. 19 cms. 16 fols.). También figura en la portada que el sermón fue dedicado a D. Gonzalo de Campo, Arcediano de Niebla y que lo mandó imprimir el Bachiller Sebastián Tovar de la Plata, presbítero.

PORCEL DE MEDINA, Juan Bautista. En la Bib. Universitaria de Sevilla, 148/36(2), hemos examinado su obra:

Instrvcion de penitentes para saber confessarse. Con singvlares auisos para todo genero y estado de personas, conforme las condiciones de la buena Confession... (Sevilla, Simón Fajardo, 1626. 13 cms. 12 hs +120 fols.).

Esta obra, en tan curioso formato, no la encontramos registrada en los catálogos bibliográficos ni por Méndez Bejarano. ¿Podría ser la misma que cita éste y Nicolás Antonio ${ }^{27}$ sin indicar número de fols. o págs., Grano de oro, modo de confesar bien, con un examen de conciencia, impresa en Sevilla en 1634 y de la que no se conoce ejemplar conservado?

ROBLES, Juan de. A pesar de no contar con datos fehacientes, tanto Méndez Bejarano como otros biógrafos anteriores, se inclinan a suponerlo sevillano; sin duda por su vinculación con la capital andaluza. No obstante, todo parece apuntar que nació en San Juan del Puerto y así lo considera Rodríguez Marín ${ }^{28}$. Sabemos que se está elaborando una tesis doctoral de la obra de Juan de Robles El culto sevillano y suponemos que se nos aclararán muchos puntos también sobre su biografía.

SANTA MARÍA, Juan de. Poco sabemos de él, únicamente que fue Secretario del Consulado de Sevilla y que participó en dos certámenes poéticos en 1651 y 1663 celebrados en Granada y Sevilla respectivamente ${ }^{29}$. Méndez Bejarano no lo registra; sí a un dominico del mismo nombre.

SOTO, Francisco de. Amplia e interesante producción nos ha quedado de este jesuita nacido en Marchena. A ella hemos de añadir una pieza menor no registrada, se trata de la siguiente:

Sermon de las llagas del seraphico Padre S. Francisco. Predicado en su casa grande de Sevilla. Por... (Sevilla, Francisco de Lyra, 1624. 20 cms. 12 fols.). Fue tam-

27 Bibliotheca Hispana Nova (Madrid: Joaquín de Ibarra y sucesores, 17 83-1788), 2 vols., I, pág. 652.

28 Nuevos datos..., op. cit., pág. 405.

29 Vid. José Simón Díaz y Luciana Calvo Ramos «Siglos de Oro: Índice de Juntas Poéticas» en Cuadernos Bibliográficos, 5 (n. monográfico), C.S.I.C., 1962, pág. 96. 
bién impreso, y de ello tampoco hay cita, en 1625 por Luis Estupiñán en Sevilla. Hay ejemplares de los dos textos en la Bib. Universitaria de Sevilla con las signaturas 113/84(37 y 113/78(10) respectivamente.

TORRES Y SALTO, Baltasar de. Aunque Méndez Bejarano se extiende en su biografía, no lo hace en cambio con su obra. Esta se encuentra recogida por Palau (XXIII, 337.270-72), pero como es habitual sin señalización de ejemplares. En la Bib. Universitaria de Sevilla, con las signaturas 113/74(16) y 111/43(13), se hallan:

Sermon... a las honras i oficios fvnebres del gran monarca... Phelipe III... Qve celebraron los dos Cabildos... de... Badajoz en la santa Iglesia Cathedral... (Sevilla, Juan Serrano de Vargas, 1621. 20 cms. 4 hs. + 40 fols.).

Discvrso de los Santos de la Santa Iglesia Metropolitana de Sevilla... (Sevilla, Francisco de Lyra, 1624. 20 cms. 20 fols.).

URTEAGA, Pedro de. Con referencia a Nicolás Antonio, Escudero cita un sermón de este padre de la Compañía de Jesús pero no vio ejemplar. Existe en la Bib. Universitaria de Sevilla (111/54(28)):

Sermon que predico el Padre - Preposito de la Casa Professa de la Compañiáa de IESVS. A las Honras que hizo la gran ciudad de Seuilla al Catolico Rey Don Felipe III. N. S. que esté en el cielo, en tres de Iunio. (Sevilla, Alonso Rodríguez Gamarra, 1621. 19 cms. 2 hs. + 24 fols.).

Palau (XXIV, 346.133) a su vez fecha la impresión en 1623. Estimamos que más que una nueva edición, se trata de un error de éste.

VENEGAS DE COLOMBRES, Francisco. Damos la noticia de una obra suya no recogida en los repertorios bibliográficos:

Declaración de el derecho que tiene N. Padre San Pedro Nolasco. Para que qualquier Iglesia, o Obispado pueda, libre, y voluntariamente admitir su rezo... (Sevilla, Juan Gómez de Blas, 1644. 20 cms. ¿8 fols.?). Sevilla Bib. Universitaria, 110/58(10). Incompleta. 
